\title{
Anuria súbita en paciente monorreno secundaria a migración de endoprótesis aórtica
}

\author{
S. Capdevila Querol, R. Gutiérrez Del Pozo, A. Franco De Castro, A. Aguilar, D. Truhán Cacho, \\ J.B. Alcover García
}

Servicio de Urología y Unidad de Trasplante Renal. Hospital Clínico y Provincial de Barcelona.

Actas Urol Esp 2005; 29 (5): 526-528

\section{RESUMEN}

\section{ANURIA SÚBITA EN PACIENTE MONORRENO SECUNDARIA A MIGRACIÓN DE ENDOPRÓTESIS AÓRTICA}

El manejo percutáneo de los aneurismas de aorta infrarrenal es una técnica menos agresiva, pero no está exenta de riesgos. La migración craneal de las endoprótesis es un proceso infrecuente en dicha técnica. Ante una revascularización renal izquierda de urgencia en que no se pueda tener acceso a una vía anterior o la posibilidad de un autotrasplante, una buena alternativa es la realización de un shunt esplenorrenal mediante un acceso lumbar.

Palabras clave: Revascularización renal urgente. Aneurisma de aorta infrarrenal. Shunt esplenorrenal.

\section{ABSTRACT}

SUDDEN ANURIA SECONDARY TO MIGRATION OF AORTIC STENT IN A SINGLE KIDNEY PATIENT

Percutaneous acces to manage infrarenal aortic aneurysm is a less aggressive technique, but it's not entirely risk free. The migration of stents isn't a frequent complication in that percutaneous technique. Urgent left renal revascularition, when anterior approach or autologous transplantation is not possible, is feasibily by a splenorenal shunt through a lumbar approach.

Key words: Urgent renal revascularition. Infrarenal aortic aneurysm. Splenorenal shunt.

$\mathrm{E}$ progresivo envejecimiento de la población, con las enfermedades asociadas que ello conlleva, supone un incremento en la morbimortalidad de la cirugía electiva de los aneurismas de aorta infrarrenal .En la mayoría de centros con experiencia, dicha cirugía reporta una tasa de mortalidad inferior al $5 \%^{1}$.

Parodi $^{2}$ en 1991, propuso una nueva técnica para el manejo de esta patología; la exclusión del saco aneurismático mediante la colocación percutánea de una endoprótesis aórtica. Aunque menos agresiva, dicha técnica no esta exenta de riesgos.

\section{CASO CLÍNICO}

Paciente de 72 años que ingresa para cirugía electiva de aneurisma de aorta abdominal infrarre- nal y de arteria hipogástrica derecha. Como antecedentes personales destaca nefrectomía derecha en 1983 por carcinoma de células renales, HTA controlada con amlodipino y IAM inferolateral antiguo con cardiopatía silente. El DIVAS aprecia una ectasia aórtica difusa y una dilatación aneurismática fusiforme cuyo cuello se sitúa a $17 \mathrm{~mm}$ de la arteria renal izquierda, con trombo mural de $7 \mathrm{~mm}$ en su interior y un calibre de luz máximo de $35 \mathrm{~mm}$ a nivel de arteria mesentérica inferior. La dilatación aneurismática afecta a ambas arterias iliacas primitivas, y se objetiva un segundo aneurisma fusiforme de $13 \mathrm{~mm}$ en arteria hipogástrica derecha. Se confirma permeabilidad de eje celíaco, arteria renal izquierda, mesentérica superior y oclusión de arteria mesentérica inferior. 
El paciente es intervenido colocándose endoprótesis aórtica (Quantum Cordis ${ }^{\circledR}$ ) 36-12 con extensión a ambas arterias ilíacas y embolización de arteria hipogástrica derecha. La arteriografia renal de control fue normal.

A las $2 \mathrm{~h}$ de la intervención se aprecia anuria de instauración súbita, sin mejoría con perfusión de diuréticos. La arteriografía urgente $(6 \mathrm{~h}$ postoperatorias) muestra oclusión total del óstium de la arteria renal izquierda por migración craneal de la endoprótesis. Se decide cirugía de revascularización urgente mediante shunt esplenorrenal por lumbotomia posterolateral izquierda a nivel $\mathrm{XII}^{\mathrm{a}}$ costilla. Se procede a disección de pedículo renal izquierdo ( 1 arteria, 1 vena) reperfusión renal mediante ringer lactato a $4 \mathrm{C}$ y anastomosis término-terminal con la arteria esplénica (puntos discontinuos de Prolene ${ }^{\circledR}$ 6/0). La reperfusión del riñón se procedió a las $12 \mathrm{~h}$ post instauración de la anuria, recuperándose progresivamente color y consistencia, manteniendo la anastomosis un buen pulso.

La diuresis espontánea reapareció a las 48 h, necesitando previamente una sesión de hemodiálisis por hiperpotasemia y acidosis. En la Angio RMN de control (Figs. 1 y 2) (2 meses) se demuestra la permeabilidad de la endoprótesis, la ausen-

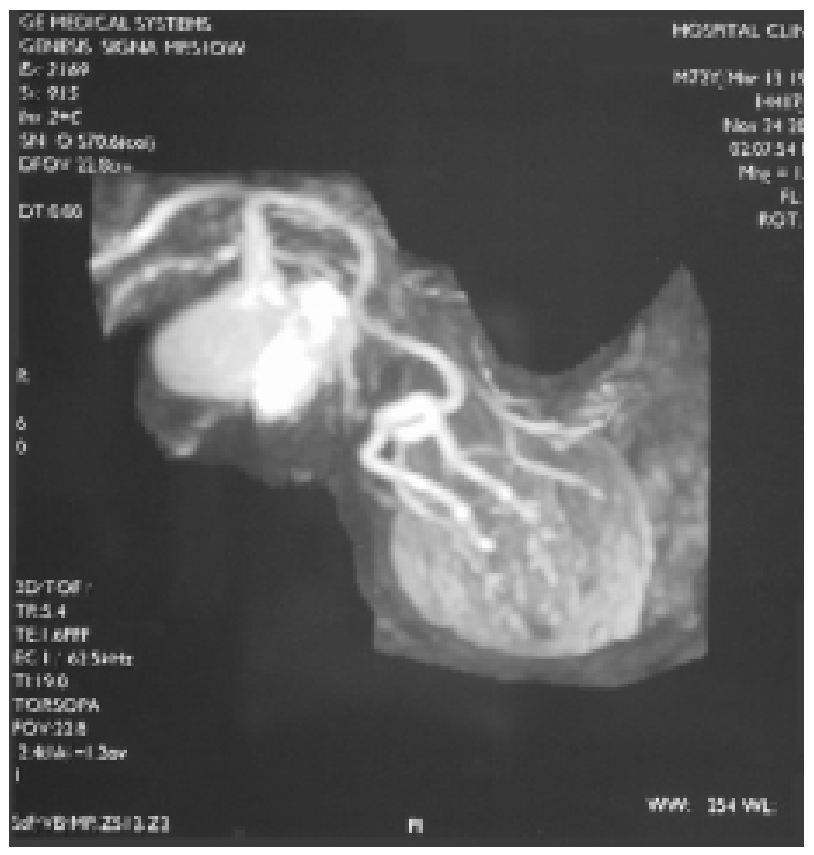

FIGURA 1. Angio RMN. Anastomosis esplenorenal, corte sagital a nivel de tronco celiaco.

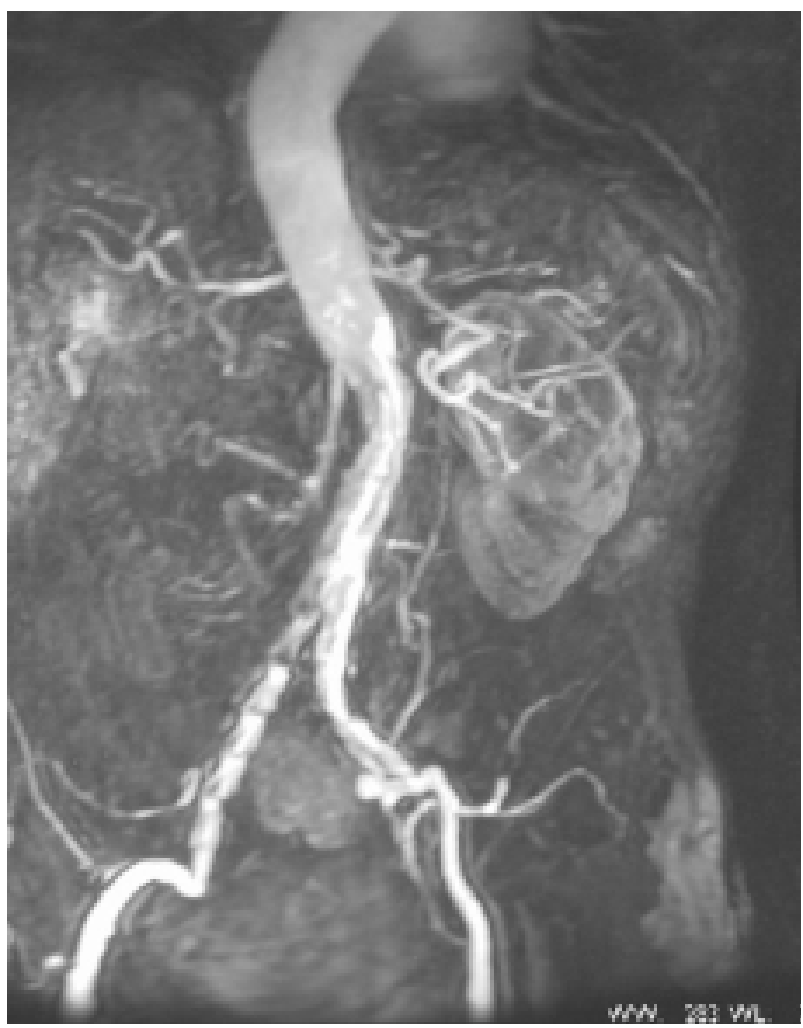

FIGURA 2. Angio RMN. Anastomosis esplenorenal, corte longitudinal.

cia de endofugas y de estenosis en la anastomosis esplenorrenal En la actualidad el paciente sigue con tensiones bien controladas con amlodipino y con cifra de creatinina de $1,4 \mathrm{mg} / \mathrm{dl}$.

\section{DISCUSIÓN}

La migración de la endoprótesis no es una complicación frecuente en el manejo percutáneo de los aneurimas de aorta infrarrenal. Longitud $\geq 15 \mathrm{~mm}$, diámetro $\leq 28 \mathrm{mmm}$, angulación $\leq 60^{\circ}$, forma no cónica; son las características del cuello del aneurisma que condicionan el anclaje superior de la endoprótesis ${ }^{3}$.

En este caso, la patología vascular aortoiliaca condicionó la revascularización renal urgente, dado que no era posible la realización de un autotrasplante o un abordaje anterior para un shunt esplenorrenal.

Gil Vernet ${ }^{4,5}$ en 1978 propuso un nuevo acceso a los vasos esplénicos mediante lumbotomia posterolateral izquierda, que permitió en años posteriores la realización del trasplante renal ortotópico. 
La migración de la endoprótesis es un proceso infrecuente y lo que hace excepcional este caso es que se tratara de un paciente monorreno izquierdo que permitió una vía de acceso brillante para solucionar el problema.

\section{REFERENCIAS}

1. Kantz DJ, Stanley JC, Zelenock GB. Operative mortality rates for intact and ruptured abdominal aortic aneuryss in Michigan: An eleven - year state .wide experience. J Vasc Surg 1994;19:804-815.

2. Parodi JC, Palmaz JC, Baronce HD. Trasfemoral intraluminal graft implantation for abdominal aortic aneurysms. Ann Vasc Surg 1991 Bov; 5(6):491-499.
3. Ohki T, Veith FJ. Patient selection for endovascular repair of abdominal aortic aneurysms: Changing the Threshld for Intervention. Sem Vasc Surg 1999;3:226-234.

4. Gil-Vernet JM, Caralps A, Ruano D. New approach to the explenic vessels. J Urol 1978 Mar;119(3):313-315.

5. Gil-Vernet JM, Gil-Vernet A, Caralps A, et al. Orthotopic renal transplant and results in 139 consecutive cases. $J$ Urol 1989 Aug;142(2 Pt 1):248-252.

Dr. S. Capdevila Querol

Servicio de Urología y Unidad de Trasplante Renal Hospital Clínic i Provincial

C/ Villaroel 170 - 08036 Barcelona

e-mail: scapdevi@operamail.com

(Trabajo recibido el 2 septiembre 2004) 